\title{
A novel AIFM1 mutation expands the phenotype to an infantile motor neuron disease
}

\author{
Daria Diodato ${ }^{1}$, Giorgio Tasca ${ }^{1}$, Daniela Verrigni ${ }^{1}$, Adele D’Amico ${ }^{1}$, Teresa Rizza ${ }^{1}$, Giulia Tozzi ${ }^{1}$, \\ Diego Martinelli ${ }^{2}$, Margherita Verardo ${ }^{1}$, Federica Invernizzi ${ }^{3}$, Alessia Nasca ${ }^{3}$, Emanuele Bellacchio ${ }^{4}$, \\ Daniele Ghezzi ${ }^{3}$, Fiorella Piemonte ${ }^{1}$, Carlo Dionisi-Vici ${ }^{2}$, Rosalba Carrozzo ${ }^{1}$ and Enrico Bertini ${ }^{\star, 1}$
}

\begin{abstract}
AIFM1 is a gene located on the $\mathrm{X}$ chromosome, coding for AIF (Apoptosis-Inducing Factor), a mitochondrial flavoprotein involved in caspase-independent cell death. AIFM1 mutations have been associated with different clinical phenotypes: a severe infantile encephalopathy with combined oxidative phosphorylation deficiency and the Cowchock syndrome, an X-linked Charcot-MarieTooth disease (CMTX4) with axonal sensorimotor neuropathy, deafness and cognitive impairment. In two male cousins with earlyonset mitochondrial encephalopathy and cytochrome $c$ oxidase (COX) deficiency, we identified a novel AIFM1 mutation. Muscle biopsies and electromyography in both patients showed signs of severe denervation. Our patients manifested a phenotype that included signs of both cortical and motor neuron involvement. These observations emphasize the role of AIF in the development and function of neurons.
\end{abstract}

European Journal of Human Genetics (2016) 24, 463-466; doi:10.1038/ejhg.2015.141; published online 15 July 2015

\section{INTRODUCTION}

The AIFM1 gene (OMIM $\left.{ }^{*} 300169\right)$ is located on chromosome $\mathrm{X}$ and encodes for AIF (Apoptosis-Inducing Factor), a mitochondrial flavoprotein involved in caspase-independent cell death. AIFM1 mutations have been reported to be associated with two main clinical phenotypes: a severe, early-onset mitochondrial encephalopathy with combined oxidative phosphorylation deficiency (COXPD6; MIM \#300816), with or without ventriculomegaly, ${ }^{1,2}$ and the Cowchock syndrome or CMTX4 (MIM \#310490), an X-linked axonal motor and sensory neuropathy associated with deafness and cognitive impairment. The Cowchock syndrome was first described in $1986^{3}$ and recently related to an AIFM1 mutation. ${ }^{4}$ Very recently, an additional AIFM1 mutation was found in a patient with early-onset ataxia, hearing loss and late-onset myopathy with progressive external ophthalmoplegia (PEO). ${ }^{5}$

In this report we describe the clinical, histochemical and biochemical findings in two cousins harboring a novel AIFM1 mutation and showing myopathological and electrophysiological signs of motor neuron involvement, in addition to severe encephalopathy with ventriculomegaly that has already been described.

\section{MATERIALS AND METHODS}

The study was approved by the Children Research Hospital 'Bambino Gesù' ethics board. Informed consent for biochemical and genetic studies was obtained from patients' caregivers.

\section{Patients}

P1 and P2, born from two sisters of a large Italian family (Figure 1a), came to our attention for a phenotype characterized by perinatal onset of marked hypotonia with proximal muscle weakness, resembling a spinal muscular atrophy (SMA) phenotype; during the first 2 months of life both babies showed rapidly progressive involvement of arms and legs with absent deep tendon reflexes, associated with global developmental delay. P1 presented with respiratory distress at birth that required tracheostomy at 3 months of age; because of severe swallowing difficulties, he underwent percutaneous enterogastrotomy (PEG) in the same period of life. P2 had a slightly milder disease course as he developed progressive respiratory and swallowing difficulties at 5 months, and then required tracheostomy and PEG at 6 months. Both babies similarly presented poor eye contact and continuous involuntary movements of small amplitude in the hands and feet. EEG showed multifocal epileptic abnormalities in central, parietal and occipital regions; they also had abnormal visual- and brainstem-evoked potentials. In P1 (Figure 1b) and P2 (Figure 1c) brain MRI displayed a frontotemporal atrophy and widening of subarachnoid spaces of the lateral ventricles, with a lactate peak at spectroscopy. Plasma lactate was mildly increased (P1: $2.4 \mathrm{mmol} / \mathrm{l} ; \mathrm{P} 2$ : $3.3 \mathrm{mmol} / \mathrm{l} ; \mathrm{nv}<2 \mathrm{mmol} / \mathrm{l})$. Nerve conduction studies in P1 showed signs of motor axonal neuropathy, associated with signs of active denervation (fibrillation potentials and positive sharp waves) at EMG.

Histochemical and biochemical analyses

Quadricep muscle and needle skin biopsies were obtained from P1 and P2. Cryostatic cross-sections were processed according to standard histochemical procedures. Mitochondrial respiratory chain (MRC) complex activities were measured using standard spectrophotometric methods ${ }^{6}$ in muscle homogenate and digitonin-treated skin fibroblasts, and normalized to citrate synthase activity, an index of mitochondrial content in the analyzed specimens.

\footnotetext{
${ }^{1}$ Neuromuscular and Neurodegenerative Diseases Unit, Children Research Hospital Bambino Gesù, Rome, Italy; ${ }^{2}$ Division of Metabolism, Children Research Hospital Bambino Gesù, Rome, Italy; ${ }^{3}$ Molecular Neurogenetics Unit, Foundation IRCCS Neurological Institute C, Besta, Italy; ${ }^{4}$ Research Laboratories, Children Research Hospital Bambino Gesù, Rome, Italy

${ }^{*}$ Correspondence: Dr E Bertini, Neuromuscular and Neurodegenerative Diseases Unit, Children Research Hospital Bambino Gesù, Viale San Paolo 15, Rome 00146, Italy. Tel: +39 066859 2105; Fax: +39 066859 2024; E-mail: enricosilvio.bertini@opbg.net

Received 16 February 2015; revised 18 May 2015; accepted 27 May 2015; published online 15 July 2015
} 
a
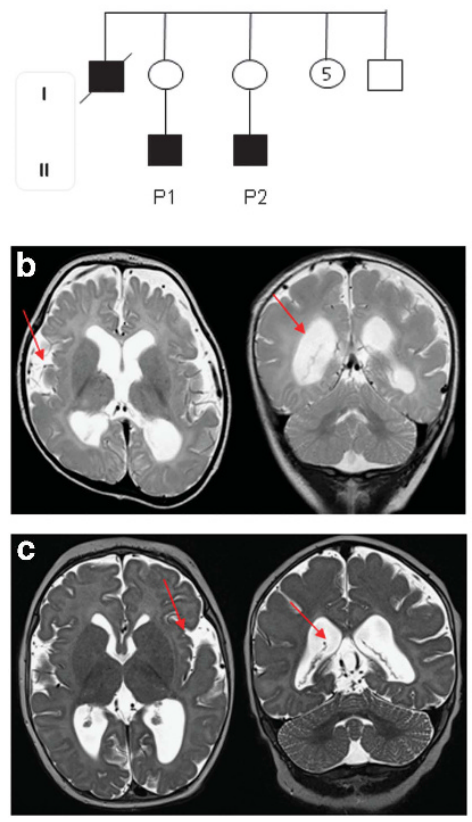
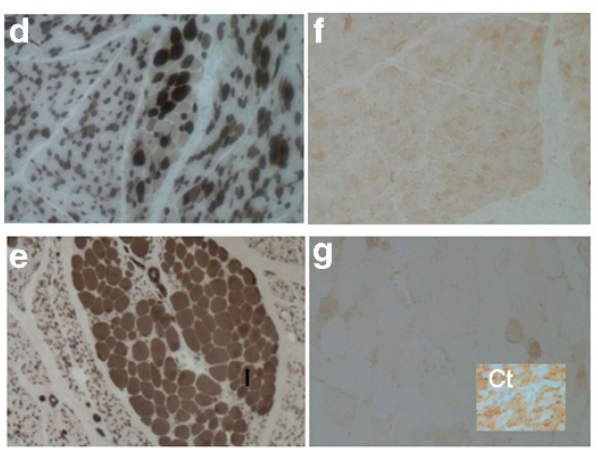

h

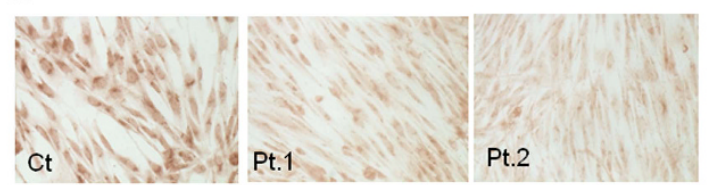

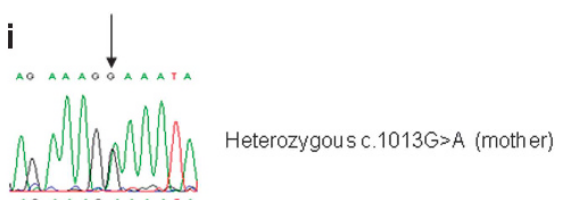
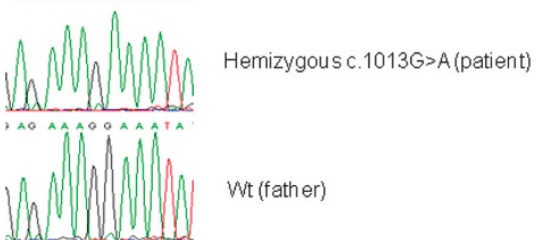

Wt (father) j

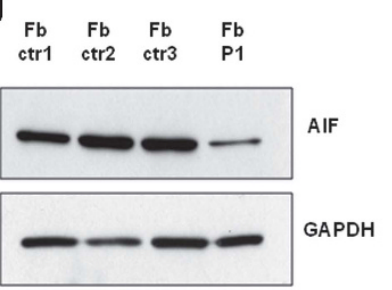

Figure 1 (a)The family tree shows the probands in the second line, and seven sisters and two brothers (one deceased) in the first line. (b and $\mathbf{c}$ ) MRI in P1 and P2. The T2-weight axial and coronal images show frontotemporal atrophy (left arrow) and widening of subarachnoid spaces of the lateral ventricles (right arrow). (d-g) Morphological analysis of muscle biopsies from P1 (upper images) and P2 (lower images). The ATP-ase staining (left side of the panel) shows neurogenic features, whereas the cytochrome $c$ oxidase staining (right side) shows a decrease in the histochemical reaction in both patients. Inset shows the reaction in an age-matched control biopsy. (h) The cytochrome $c$ oxidase staining shows a decreased reaction in both patients' fibroblasts compared with a control line (left side). (i) The electropherogram of AIFM1 in P1 and his mother, compared with a wild-type sequence. The arrow indicates the position of the nucleotide change identified in our patients. (j) Western blot analysis in controls and patient's fibroblasts using AIF and GAPDH antibodies: the patient's sample shows a clear reduction in the AIF amount compared with the control lines.

\section{Mutational analysis}

Total genomic DNA was extracted by standard methods from cultured skin fibroblasts or peripheral blood of patients and family members. The coding exons and exon-intron boundaries of the AIFM1 gene (RefSeq NM_004208.3) were PCR-amplified using specific primers, cycle sequenced using BigDye chemistry 3.1 and were run on an $\mathrm{ABI}$ 3130XL automatic sequencer (Applied Biosystems, Life Technologies, Waltham, MA, USA). The identified variant has been submitted to LSDB (http://www.LOVD.nl/AIFM1; ID 65306).

\section{Western blot analysis}

Fibroblasts were trypsinized, centrifuged at $1000 \mathrm{~g}$ for $5 \mathrm{~min}$, and solubilized in RIPA buffer with protease inhibitors. Proteins $(50 \mu \mathrm{g})$ were loaded for each sample in $10 \%$ denaturing sodium-dodecyl sulfate polyacrylamide gel electrophoresis (SDS-PAGE). Immunoblot analysis was performed with the ECL-chemiluminescence kit (GE Healthcare, Little Chalfont, Buckinghamshire, UK) according to the manufacturer's protocol. A rabbit polyclonal anti-AIF antibody (Millipore, Billerica, MA, USA) and a mouse monoclonal antiGAPDH (Millipore) were used.

\section{RESULTS}

Muscle pathology in both patients showed neurogenic changes (Figures $1 \mathrm{~d}$ and e), which were particularly evident in P2 where the presence of large groups of markedly atrophic muscle fibers, clusters of hypertrophic fibers and type groupings at ATPase staining closely resembled the histological pattern of SMA (Figure 1e). The histochemical staining for cytochrome $c$ oxidase (COX) was performed in fibroblasts and muscles, revealing COX deficiency in both patients
(Figure 1f-h). Spectrophotometric assay of MRC complex activities displayed an isolated reduction of COX (50\% of control mean) in P1 muscle; unfortunately, the amount of $\mathrm{P} 2$ muscle was not adequate to perform this assay. Biochemical analyses in P1 and P2 fibroblasts showed a partial decrease in COX activity of 20 and 45\%, respectively. Other MRC complex activities were in the control range in patients' fibroblasts and muscle. Sanger sequencing of AIFM1 revealed a novel nucleotide substitution (c.1013G > A; Figure 1i), hemizygous in both $\mathrm{P} 1$ and $\mathrm{P} 2$ and heterozygous in their mothers. This missense change p.(Gly338Glu) is predicted to affect protein function by different softwares (Polyphen2, SIFT, Mutation Taster). This variant has not been reported in databases of single-nucleotide polymorphisms including dbSNP build 142 and Exome Aggregation Consortium (ExAC; http://exac.broadinstitute.org).

The deleterious effect of the identified variant was confirmed using western blot analysis, which showed strongly decreased amount of AIF protein in P1 fibroblasts when compared with controls (Figure 11).

The p.(Gly338Glu) substitution replaces a highly conserved glycine (Figure 2a). In the AIF protein structure Gly338 presents only minimal exposure to the solvent; therefore, the replacement of this neutral and tiny residue with a glutamic acid implies structural and electrostatic changes. These changes may have functional consequences because they involve residues that interact with the regulatory 510-550 peptide region, whose conformation is regulated by AIF redox state changes and thought to determine the protein localization, lifetime, posttranslational modifications and interactions with other proteins and DNA. ${ }^{7}$ In fact, upon reduction with $\mathrm{NADH}$ the 510-560 peptide undergoes major dislocation, and despite being largely unstructured, a portion of this peptide results anchored nearby the site of the 
a

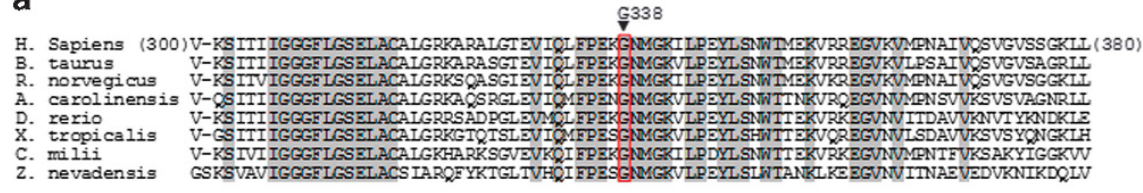

b

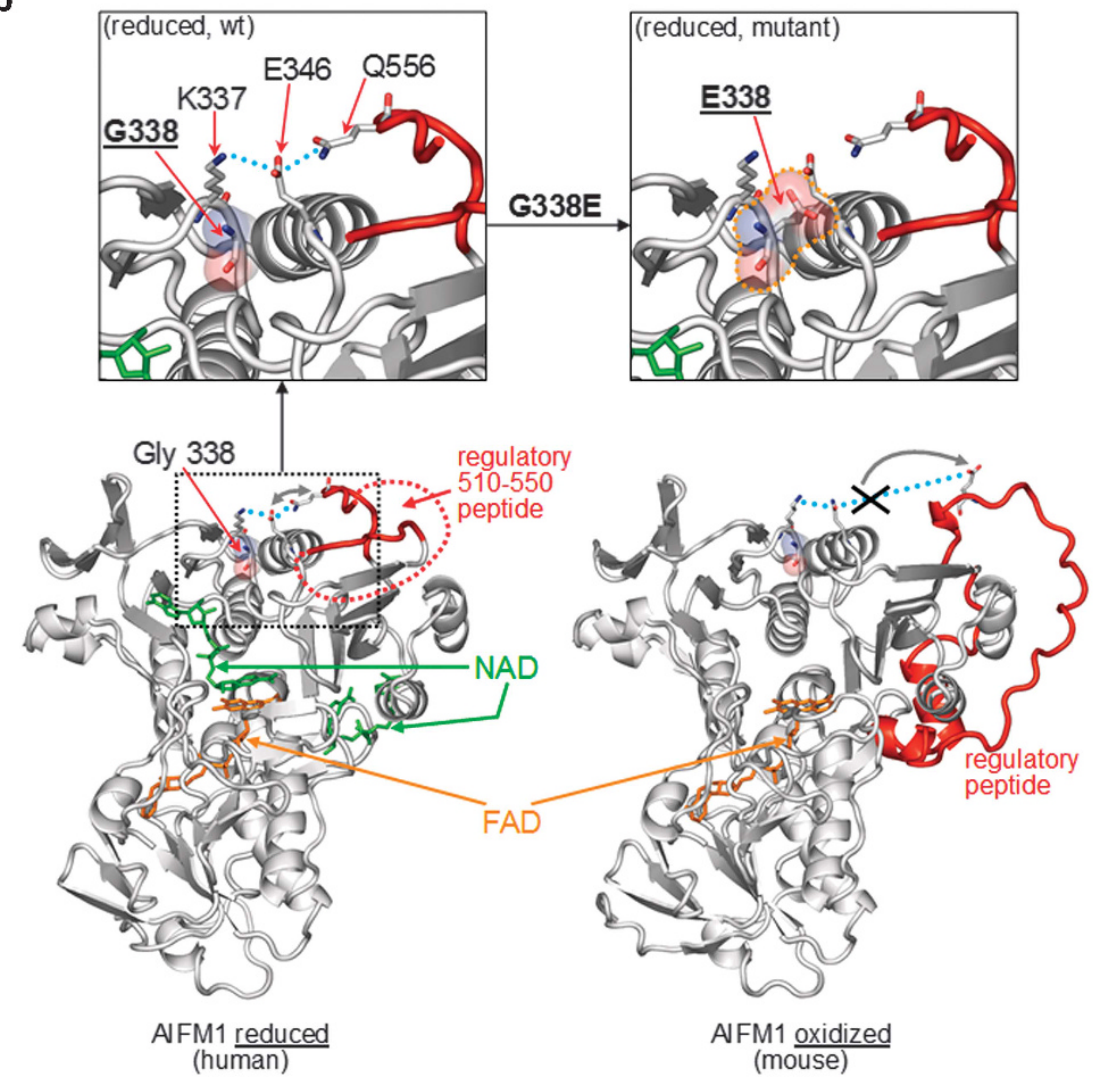

Figure 2 (a) Multiple sequence alignment of AIFM1 protein across species. Gly 338 in the human protein is indicated by the black triangle. Grayed columns indicate invariant residues. (b) Molecular structures of reduced human AIFM1 (Protein Data Bank, PDB, 4BUR) and oxidized mouse AIFM1 (PDB 1GV4). G338, K337, E346 and Q556 in the reduced human protein, and the corresponding residues in the oxidized mouse protein are shown as sticks. For enhanced clarity, the site of the G338E mutation is also highlighted by a transparent surface. The magnified view of the region around Gly 338 for the reduced human protein is shown together with an in silico Gly 338 to Glu replacement and optimization of Glu 338 side chain to highlight the alterations that can be induced by the mutation on the interactions (indicated by azure dots) involving nearby residues. In particular, it can be noticed that these interactions contribute to the folding of the regulatory 510-560 peptide exclusively in the reduced form of the protein (please compare the structures of the reduced and oxidized AIFM1). The 510-560 peptide is colored in red (the red dotted line in the reduced human AIFM1 represents disordered portion of the peptide). Molecular structures were rendered with PyMOL.

p.(Gly338Glu) change by a network of interactions involving Lys337 (adjacent to Gly338), Glu346 and Gln556 (belonging to the regulatory peptide; Figure $2 \mathrm{~b}$ ). On the other hand, in the oxidized AIF the 510-550 peptide is far away from the site of the p.(Gly338Glu) change. It is worth to mention that Lys337 undergoes acetylation in human and murine AIF, ${ }^{8,9}$ allowing to hypothesize that this post-translational modification may have a role in regulating the conformation and function of the 510-550 peptide. The p.(Gly338Glu) substitution falls nearby residues that bind the NAD molecule involved in the formation of the $\mathrm{FADH}_{2}$ NAD charge-transfer complex; thus, it may also influence this interaction.

\section{DISCUSSION}

AIF is a mitochondrial FAD-dependent protein involved in apoptosis, mitochondrial function, ROS production and neurodegeneration. ${ }^{10}$ Patients with mitochondrial encephalomyopathy first described by Ghezzi et al. ${ }^{1}$ harbored a hemizygous trinucleotide deletion (c.603_605del) in the AIFM1 gene predicting the ablation of an arginine at position 201 (p.Arg201del). In muscle of these patients there was a clear histochemical COX deficiency, together with a combined biochemical defect of MRC complex activities; however, no signs of denervation were observed. In vitro studies suggested that the p.Arg201 deletion affects both redox and DNA-binding properties of AIF protein. ${ }^{1}$

Patients with Cowchock syndrome were found to harbor the AIFM1 c.1478A > T mutation, which predicts a new p.Glu493Val change: ${ }^{4}$ these patients displayed modest signs of neurogenic atrophy at muscle biopsy, but did not show histochemical COX deficiency or MRC defects at biochemical analysis. ${ }^{4}$ Authors concluded that the mildest phenotype caused by the p.Glu493Val change was the consequence of a pathogenic mechanism related to apoptotic dysfunction and independent from oxidative phosphorylation.

A different hemizygous mutation in AIFM1 gene, the c.923G > A (p.Gly308Glu), was identified in three siblings with severe infantile 
encephalomyopathy and combined oxidative phosphorylation defect. The p.Gly308Glu substitution affects a glycine in the region of binding of the $\mathrm{FADH}_{2}-\mathrm{NAD}$ charge-transfer complex. This region is characterized by highly tuned interactions with the ligands necessary for the redox activity of the protein. ${ }^{7}$ The replacement of glycine 308 with a glutamic acid residue cannot occur without inducing important rearrangements that likely impair the catalytic function and may explain the observed severe phenotype.

Very recently Kettwig et al. ${ }^{5}$ described a young patient with a novel AIFM1 mutation, c.727G > T (p.Val243Leu), and a clinical picture characterized by progressive muscle atrophy, ataxia, hearing loss and PEO. Muscle biopsy showed unspecific myopathic changes but did not show ragged red fibers; moreover, there was a mild, diffuse reduction in COX histochemical staining. Biochemical analyses revealed a combined complex I and IV defect. This patient showed a clinical presentation intermediate between the COXPD6 and the Cowchock syndrome.

Here we describe two cousins harboring a novel AIFM1 mutation, associated with a peculiar phenotype that includes a mitochondrial encephalopathy with ventriculomegaly together with clinical, electrophysiological and myopathic signs of motor neuron involvement. Our patients were found to be hemizygous for a novel AIFM1 missense nucleotide substitution (c.1013G > A; p.Gly338Glu) that segregates in the family. COX deficiency was evident in patients' fibroblasts and muscle. Moreover, muscle biopsies disclosed a severe neurogenic pattern resembling SMA. Therefore, our patients manifest most of the previously described features associated to AIFM1 mutations; however, in addition we found clear signs of early-onset severe motor neuron involvement.

Our analysis of crystal structure of AIF protein suggests that the p.(Gly338Glu) change is likely to exert its pathogenic mechanism by destabilizing interactions involved in nearby docking of a portion of the regulatory 510-550 peptide. It must be underscored that also the already reported p.Glu493Val ${ }^{4}$ and p.Arg201del ${ }^{1}$ changes respectively produce mild and severe effects on the 510-550 peptide; however, in this case both wild-type residues contribute to the folding of this peptide specifically in the oxidized form of AIF protein. Conversely, the p.(Gly338Glu) substitution affects the regulatory peptide specifically when AIF is in the reduced state. Thus, particular functions of the regulatory peptide that are exclusively carried out when the protein is either in one or the other redox state can be selectively affected by the different amino-acidic changes, and this could explain the peculiarity of the phenotype associated with the current p.(Gly338Glu) change. Moreover, whereas the p.Arg201del does not reduce protein expression, ${ }^{1}$ the p.(Gly338Glu) change determines a severe decrease in AIF protein as also observed in samples from the patient harboring the p.Val243Leu change. ${ }^{5}$ The variable effect of different mutations on AIF protein stability and expression may contribute to the clinical variability observed in AIFM1-mutant patients.

The harlequin mutant $(\mathrm{Hq})$ mouse harbors a retroviral insertion in the $p c d 8$ gene encoding AIF, making this mouse an animal model for the AIF-related disorder; ${ }^{11}$ however, this genetic modification causes an $\sim 80-90 \%$ global AIF reduction rather than a change in the protein sequence. AIF reduction does not cause embryonic lethality and the Hq mice present slow, progressive neurodegeneration affecting the cerebellar, thalamic, striatal and cortical regions resulting in ataxia and loss of vision; this is associated with a reduced complex I expression in these brain areas. ${ }^{11,12}$ The role of AIF in programmed cell death, oxidative damage and neurodegeneration has been partially investigated, ${ }^{10,13}$ whereas its role in neuron development and the relation with the described clinical phenotypes have not been so far elucidated. On the basis of current knowledge and our patients' findings, we speculate that AIFM1 mutations are responsible for a diffuse neuronal damage, including both cortical neurons in the brain and motor neurons in the spinal cord. The array of mutations in AIFM1 seems to be associated with a broad range of clinical presentations. Phenotypical variability such as motor neuron involvement rather than oxidative dysfunction or a global damage might depend on different levels of AIF reduction, its relation with thresholddependent multiple functions of this protein or, as we explained here, by different mutational effects on AIF global structure and interactions. Further molecular and modeling studies are therefore needed to elucidate the AIF role in neuronal development and function.

\section{CONFLICT OF INTEREST}

The authors declare no conflict of interest.

\section{ACKNOWLEDGEMENTS}

We thank the families for their collaboration.

\section{AUTHOR CONTRIBUTIONS}

GT, MV and TR performed histochemical studies, GT performed biochemical analyses, DV and FI performed mutational analyses, AN performed WB analysis of AIF, AD, DM, CDV visited patients, supplied clinical details and critically reviewed the manuscript, DG, FP, RC critically analyzed data and reviewed the manuscript, EB performed the analysis of AIFM1 gene structure and function, DD and ESB designed the study, wrote the draft and revised it critically. All authors read and approved the manuscript.

1 Ghezzi D, Sevrioukova I, Invernizzi F et al: Severe X-linked mitochondrial encephalomyopathy associated with a mutation in apoptosis-inducing factor. Am J Hum Genet 2010; 86: 639-649.

2 Berger I, Ben-Neriah Z, Dor-Wolman T et al: Early prenatal ventriculomegaly due to an AIFM 1 mutation identified by linkage analysis and whole exome sequencing. Mol Genet Metab 2011; 104: 517-520.

3 Cowchock FS, Duckett SW, Streletz LJ, Graziani LJ, Jackson LG: X-linked motor-sensory neuropathy type-II with deafness and mental retardation: a new disorder. Am J Med Genet 1985; 20: 307-315.

4 Rinaldi C, Grunseich C, Sevrioukova IF et al: Cowchock syndrome is associated with a mutation in apoptosis-inducing factor. Am J Hum Genet 2012; 91: 1095-1102.

5 Kettwig M, Schubach M, Zimmermann FA et al: From ventriculomegaly to severe muscular atrophy: expansion of the clinical spectrum related to mutations in AIFM1. Mitochondrion 2015; 21C: 12-18.

6 Bugiani M, Invernizzi F, Alberio S et al: Clinical and molecular findings in children with complex I deficiency. Biochim Biophys Acta 2004; 1659: 136-147.

7 Sevrioukova IF: Redox-linked conformational dynamics in apoptosis-inducing factor. J Mol Biol 2009; 390: 924-938.

8 Lundby A, Lage K, Weinert BT et al: Proteomic analysis of lysine acetylation sites in rat tissues reveals organ specificity and subcellular patterns. Cell Rep 2012; 2: 419-431.

9 Weinert BT, Schölz C, Wagner SA et al: Lysine succinylation is a frequently occurring modification in prokaryotes and eukaryotes and extensively overlaps with acetylation. Cell Rep 2013; 4: 842-851.

10 Polster BM: AIF, reactive oxygen species, and neurodegeneration: a 'complex' problem. Neurochem Int 2013; 62: 695-702.

11 Klein JA, Longo-Guess CM, Rossmann MP et al: The harlequin mouse mutation downregulates apoptosis-inducing factor. Nature 2002; 419: 367-374.

12 El Ghouzzi V, Csaba Z, Olivier P et al: Apoptosis-inducing factor deficiency induces early mitochondrial degeneration in brain followed by progressive multifocal neuropathology. J Neuropathol Esxp Neurol 2007; 66: 838-847.

13 Joza N, Susin SA, Daugas E et al: Essential role of the mitochondrial apoptosis-inducing factor in programmed cell death. Nature 2001; 410: 549-554. 